\title{
Effects of Edge Taper on Domain Wall Structure and Current-Driven Walker Breakdown in a Ferromagnetic Thin Film Wire
}

\author{
Jinshuo Zhang ${ }^{1}$, Jean Anne Currivan-Incorvia ${ }^{1,2,3}$, Larysa Tryputen ${ }^{1}$, Pin $\mathrm{Ho}^{1}$, Marc Baldo ${ }^{2}$, \\ and Caroline A. Ross ${ }^{1}$ \\ ${ }^{1}$ Department of Materials Science and Engineering, Massachusetts Institute of Technology, Cambridge, MA 02139 USA \\ ${ }^{2}$ Department of Electrical Engineering and Computer Science, Massachusetts Institute of Technology, Cambridge, MA 02139 USA \\ ${ }^{3}$ Department of Physics, Harvard University, Cambridge, MA 02138 USA
}

Received 21 Nov 2014, revised 22 Jan 2015, accepted 5 Feb 2015, published 9 Feb 2015, current version 13 Mar 2015.

Abstract-Domain walls in a ferromagnetic thin film wire with rectangular cross section adopt transverse wall (TW) or vortex wall (VW) geometries depending on the magnetic material and the wire width and thickness. However, experimentally wires can have a trapezoidal cross section if they are made by liftoff using an undercut resist profile. Micromagnetic modeling shows that tapering of the wire not only promotes the formation of a TW over a VW, but also increases the critical current value and the domain wall velocity at which Walker breakdown occurs, providing a potential route to higher speed domain wall devices.

Index Terms-Magnetodynamics, magnetic materials, spintronic materials, nanomagnetics, information storage, simulation, spin electronics.

\section{INTRODUCTION}

Ferromagnetic domain walls (DWs) in thin film wires can be translated by an electric current using spin torque transfer effects [Berger 1978, Tatara 2004, Thiaville 2007, Beach 2008, Zhang 2013] making them promising for applications in memory [Parkin 2008] and logic [Currivan 2012, Allwood 2005] devices, and spurring intense interest in their structure and behavior. In a thin film wire made from a low anisotropy ferromagnet such as Permalloy $\left(\mathrm{Ni}_{80} \mathrm{Fe}_{20}\right)$, the domain wall can adopt a transverse wall (TW) or vortex wall (VW) configuration depending on the wire width and thickness, according to both simulations and experimental observations [McMichael 1997, Kläui 2004]. The DW configuration that forms is expected to be the one that minimizes the sum of the exchange energy and magnetostatic energy, and TWs are predicted to form in thinner and narrower wires. In a rectangular cross section Permalloy wire, the phase boundary separating TW and VW configurations follows the relation $T \cdot W=C \delta^{2}$, where $T$ and $W$ are the film thickness and width, respectively, $C$ is a constant, and $\delta$ is the exchange length [McMichael 1997]. Simulations of Permalloy gives $C \approx 128$ and $\delta=4 \mathrm{~nm}$ [McMichael 1997]. A metastable asymmetric-transverse wall (ATW) state was also predicted to exist between the TW and VW regimes [Nakatani 2005]. Many studies have also shown that DWs move under the influence of a field or current with a velocity that increases until the onset of Walker breakdown (WB), an instability in the DW structure marked by periodic changes in the DW structure between TW and VW [Beach 2008]. This is particularly important in limiting the maximum operating speed of devices based on DW motion.

These studies are based on rectangular cross-sectional wires, but in practice, when wires are made by liftoff with an undercut resist profile, the wires have a trapezoidal cross section, particularly, if the film is made by a process which includes

Corresponding author: J. Zhang (zhangjs@mit.edu). Digital Object Identifier 10.1109/LMAG.2015.2402113 an oblique component of the deposition flux [Griffin 2000]. The trapezoidal geometry will lead to a change in the stray field at the tapered facet of the film compared to the rectangular case, and thus, the phase boundary between different domain wall configurations and the dynamic behavior of the wall may be expected to change. Tapering has been shown to affect resonant behavior such as edge modes [Maranville 2006]. However, the implications of tapering on domain wall structure and currentdriven dynamics have not been described. In this paper, we first demonstrate experimentally the formation of tapered edges in wires made by sputtering and liftoff. Based on the experimentally accessible geometries, we then use micromagnetic simulations to study the effect of edge taper on DW configuration and dynamic behavior.

\section{SAMPLE PREPARATION}

Sputter deposition is commonly used for thin film growth due to its control over uniformity and stoichiometry, but sidewall deposition impedes liftoff when using single layer resist. An undercut resist profile can be employed to improve edge roughness of the patterned features, using a bilayer resist, where the two layers have different solubilities in the developer after exposure, as shown in Fig. 1(a). To illustrate this, a methyl methacrylate (MMA)-polymethyl methacrylate (PMMA) bilayer resist was exposed by electron-beam lithography and developed. DC magnetron sputter deposition was used to deposit $\mathrm{Ta}(5 \mathrm{~nm}) / \mathrm{Py}(12$ $\mathrm{nm}) / \mathrm{Au}(3 \mathrm{~nm})$, with Ar pressure of $1 \mathrm{mTorr}$ and base pressure of $2 \times 10^{-8}$ Torr, followed by lift-off.

Fig. 1(b) shows an AFM image of a wire made by this method. The wire has much lower edge roughness than a wire made from single-layer resist, but it also shows a significant edge taper. The tapering slope is defined by $T:\left[\left(W_{\max }-W_{\min }\right) / 2\right]$ with $W_{\max }$ the maximum wire width and $W_{\min }$ the width of the flat top facet of the wire. Average tapering slopes of up to $\approx 10 \mathrm{~nm}$ : $500 \mathrm{~nm}=1: 50$ were produced as shown in the left panel of 


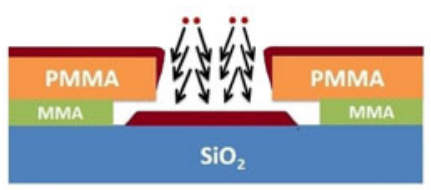

(a)

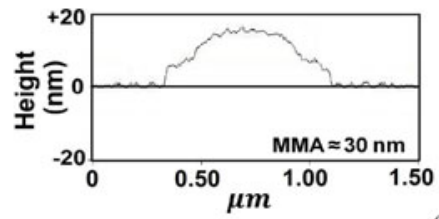

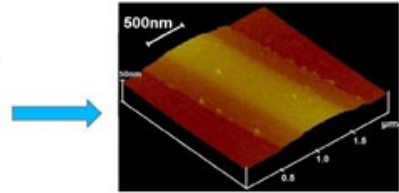

(b)

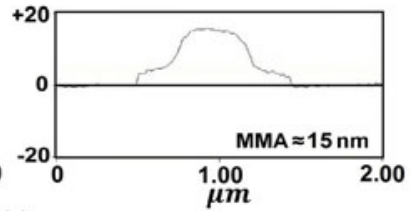

(c)

Fig. 1. (a) Schematic showing that an undercut double layer resist diminishes side-wall deposition by sputtering on the features remaining after liftoff. (b) AFM images showing good edge roughness and a tapered cross section of a wire made using an undercut resist. (c) Cross-sectional geometry of two wires made using bilayer resist. The right panel shows a wire with a thinner MMA layer.

Fig. 1(c). The samples showed a stepped cross section, and when the undercut MMA layer was thinner (15 nm instead of 30 $\mathrm{nm})$, the cross section consisted of a thicker center part on a wide, thin base. This is seen in the cross section AFM analysis in the right panel of Fig. 1(c), where the widest region of the wire was about twice that of the upper two thirds of the film and a clear step was visible. These results illustrate the formation of wires with significant tapering, whose cross section deviates significantly from a rectangular shape.

\section{MICROMAGNETIC SIMULATION}

The observed wire profile motivated our micromagnetic study of DWs in trapezoidal wires using OOMMF code [Donahue 1999], which is based on solving the Landau-Lifshitz-Gilbert equation with both adiabatic and nonadiabatic spin torque terms to include the effect of current. The simulations do not include thermal fluctuations and correspond to $0 \mathrm{~K}$. The parameters used in the simulation for Permalloy were an exchange constant $A=1.3 \times 10^{-6} \mathrm{erg} / \mathrm{cm}$, saturation magnetization $M_{s}=800$ emu/ $\mathrm{cm}^{-3}$, and Gilbert damping factor $\alpha=0.02$ [Thiaville 2007]. The magnetocrystalline anisotropy was taken as zero. The wire length was $5 \mu \mathrm{m}$ and thickness $12 \mathrm{~nm}$. The cell size used was 5 $\mathrm{nm} \times 5 \mathrm{~nm} \times 4 \mathrm{~nm}$ which is comparable to the exchange length of Permalloy. In order to simulate the tapering, the width of the wire was varied for the three layers of cells in the out-of-plane $(z)$ direction. The shape is defined by $W_{\min }$ and $W_{\max }$, the widths of the flat top section of the wire and of the base of the wire, respectively. For example, $W_{\min }=200 \mathrm{~nm}$ and $W_{\max }=300 \mathrm{~nm}$ means the top, middle, and bottom layers of cells had widths of 200 , $250\left(=W_{\text {mid }}\right)$, and $300 \mathrm{~nm}$, respectively. Finally, to model DWs in a stepped film such as in the right-hand image of Fig. 1(c), a wire with bottom layer width of $400 \mathrm{~nm}$ and middle and top layers of $200 \mathrm{~nm}$ was simulated. The tapers chosen for the model were shallow, e.g., $14^{\circ}$ for $W_{\min }=200 \mathrm{~nm}$ and $W_{\max }=300 \mathrm{~nm}$, but were still steeper than the experimentally measured average taper angles which were up to $1.2^{\circ}$.

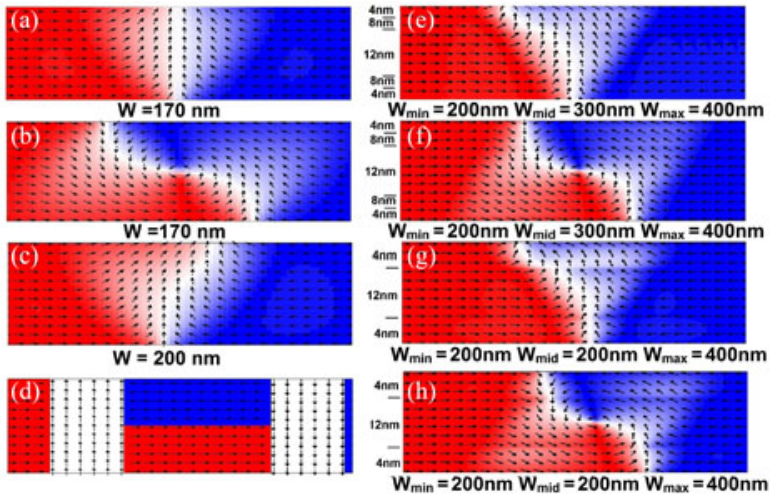

Fig. 2. (a)-(c) and (e)-(h) show DWs of different types in either a rectangular wire or a tapered wire, with the center, thick part of the wire indicated in (e)-(h). (a)-(b) TW or VW formed in a rectangular wire with $W=170 \mathrm{~nm}$. (c) ATW formed in a rectangular wire with $W=200 \mathrm{~nm}$. (d) The initial state used in the tapered wires to facilitate formation of VWs. (e)-(f) ATW or VW formed in a trapezoidal-like wire: slight discontinuity of the magnetization can be seen at the edge. (g)-(h) ATW or VW formed in a stepped wire: greater discontinuity can be observed than in (e) and (f). Arrows mark the location of the step.

To produce TWs, the magnetization in the first $2.3 \mu \mathrm{m}$ length of the wire was initialized along $+x$, where the $x$-axis is parallel to the wire, and the last $2.3 \mu \mathrm{m}$ length of the wire was initialized along $-x$. The central $0.4 \mu \mathrm{m}$ of the wire was initialized to be along $y$ (in plane transverse to the wire). In comparison, an initial configuration shown in Fig. 2(d) was used, where the magnetization was arranged to form an approximate vortex to facilitate convergence of the simulation into a $\mathrm{VW}$. After relaxing the structure from these different initial conditions, the lowest energy configuration was selected as the equilibrium configuration of the DW.

Simulations were first done on rectangular cross-sectional wires $\left(W_{\min }=W_{\max }=W\right)$. For a fixed film thickness of $12 \mathrm{~nm}$, a TW is favored when $W<170 \mathrm{~nm}$ and a VW when $W \geq 170$ $\mathrm{nm}$, corresponding to a phase boundary of $T \cdot W=128 \delta^{2}$, consistent with reported results [Donahue 1999]. In wider wires, a VW had lower energy, and simulations of TWs produced an asymmetric-transverse wall (ATW) as the metastable state instead of a symmetrical TW as shown in Fig. 2(c). This has also been observed in previous simulation reports [Nakatani 2005].

Simulations were then done on trapezoidal-like wires with the same film thickness of $12 \mathrm{~nm}$ and varying width and taper defined by $W_{\min }, W_{\text {mid }}$, and $W_{\max }$. Examples of the DW configurations are shown in Fig. 2(e)-(h). For the smoothly tapered wire of Fig. 2(e) and (f), the VW is the lowest energy configuration. The VW and TW broadly resemble those in the rectangular wire except there is a larger component of magnetization perpendicular to the wire edges in the tapered wire.

For a wire with more prominent stepped thickness profile in the right-hand image of Fig. 1(c), further distortions in the DW configuration occur. Fig. $2(\mathrm{~g})$ and $(\mathrm{h})$ shows a simulated TW and VW in a wire, where the width of the middle and top layers of the wire is the same. For both the TW and the VW, abrupt variations in the wall structure are evident at the location of the step in the wire width. 
(a)

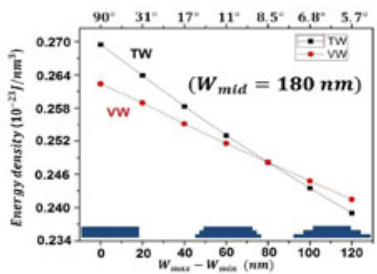

(c)

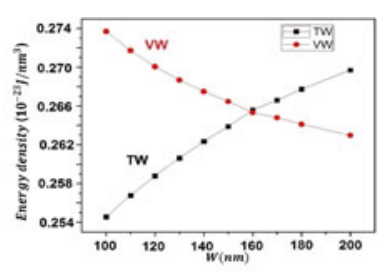

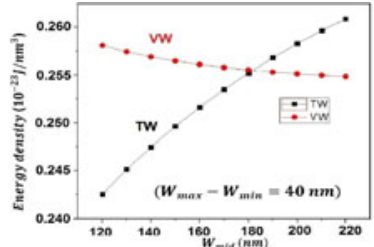

(b)

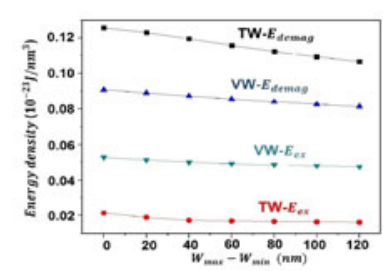

(d)
Fig. 3. (a) DW energy density dependence on rectangular wire width. (b) DW energy density in dependence on trapezoidal wire width. Energy plotted versus $W_{\text {mid }}=\left(W_{\max }+W_{\min }\right) / 2$ for a fixed taper of $W_{\max }-W_{\min }$ $=40 \mathrm{~nm}$. (c) DW energy density versus taper for fixed wire volume. Energy is plotted versus $W_{\max }-W_{\min }$. The top axis shows the corresponding taper angles. (d) DW demagnetization energy density and exchange energy density versus taper for fixed wire volume.

The total energy per unit volume, consisting of demagnetization energy and exchange energy, was calculated for different wire geometries. As shown in Fig. 3(a), in a rectangular wire, as the width $W$ increased the energy per unit volume increased for a TW, but decreased for a VW, giving a transition from TW to $\mathrm{VW}$ at a width of $W=160 \mathrm{~nm}$ in the simulation of the $12 \mathrm{~nm}$ thick NiFe wire. A similar trend was found for trapezoid wires if the taper angle was fixed by fixing $\left(W_{\max }-W_{\min }\right)$ and varying $W_{\min }$ as shown in Fig. 3(b).

However, if the volume of the wire was held constant by fixing the average width $\left(W_{\min }+W_{\max }\right) / 2$, while changing the taper (i.e., changing $W_{\max }-W_{\min }$ ), the energy per unit volume decreased with increasing taper for both TWs and VWs as shown in Fig. 3(c), which is plotted for $W_{\text {mid }}=\left(W_{\min }+W_{\max }\right) / 2=180 \mathrm{~nm}$. For this value of $W_{\text {mid }}$, the TW energy decreased faster with increasing taper than did the VW energy. This led to a transition from TW to VW, when $W_{\max }-W_{\min } \geq 80 \mathrm{~nm}$. The implication is that tapering the wire while preserving its volume promotes TWs over VWs.

This transition occurs because the demagnetization field at the edge of trapezoidal wires is affected by the taper. This can be better appreciated by plotting the demagnetization energy $E_{\text {demag }}$ and exchange energy $E_{\text {ex }}$ versus amount of taper separately for both TW or ATW and VW. In Fig. 3(d), the wall energy was calculated by subtracting the total energy for a simulation containing a DW from the total energy of the wire when it formed a single domain (ground state). A TW or ATW had a greater demagnetization energy than a VW, which is consistent with results on rectangular wires [Donahue 1999]. However, with increasing tapering, $E_{\text {demag }}$ of a TW/ATW decreased much faster than the $E_{\text {demag }}$ of a $\mathrm{VW}$, which is why tapering promotes the TW configuration.

We now consider the effect of tapering on the current-driven dynamic behavior of domain walls. A spin current in the range of 60 to $280 \mathrm{~m} / \mathrm{s}$ with $20 \mathrm{~m} / \mathrm{s}$ step size was applied, with

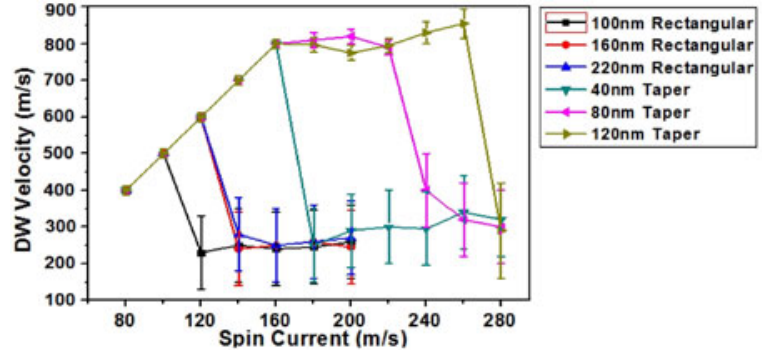

(a)

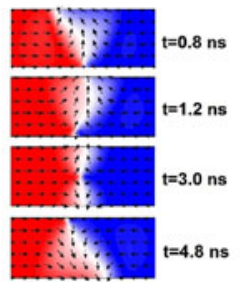

(b)

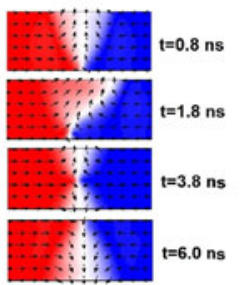

(c)

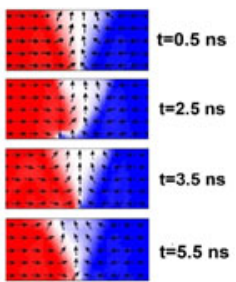

(d)
Fig. 4. (a) Domain wall velocity dependence on spin current velocity for wires with different geometries. (b) Snapshots of DW configuration during WB $(u=180 \mathrm{~m} / \mathrm{s})$ for a $160 \mathrm{~nm}$ wide rectangular wire. (c) Snapshots of DW configuration during WB $(u=180 \mathrm{~m} / \mathrm{s})$ for a $W_{\min }=140$ $\mathrm{nm}$ and $W_{\max }=180 \mathrm{~nm}$ trapezoid wire. (d) Snapshots of DW configuration during vibrational instability $(u=240 \mathrm{~m} / \mathrm{s})$ for a $W_{\min }=100 \mathrm{~nm}$ and $W_{\max }=220 \mathrm{~nm}$ trapezoid wire.

nonadiabatic parameter $\beta=0.10$. Four different geometries were compared, with thickness $12 \mathrm{~nm}$ : rectangular cross sections with $W=140,160,180,200$ and $220 \mathrm{~nm}$ in which the stable DW type changes from TW to VW with increasing width; and tapered wires of 1) $W_{\min }=140 \mathrm{~nm}, W_{\max }=180$ $\mathrm{nm}$; 2) $W_{\min }=120 \mathrm{~nm}, W_{\max }=200 \mathrm{~nm}$; and 3) $W_{\min }=100 \mathrm{~nm}$, $W_{\max }=220 \mathrm{~nm}$. These tapered wires have the same crosssectional area, and average width of $160 \mathrm{~nm}$, but different taper widths of 40,80 , and $120 \mathrm{~nm}$, respectively.

From theoretical studies [Schryer 2003, Mougin 2007], WB occurs in a rectangular wire when $(\beta-\alpha) \frac{u_{c}}{\gamma \Delta}=2 \pi \alpha M_{s} \mid N_{y}-$ $N_{z}$ l, where $u_{c}$ is the critical spin current velocity, $\gamma$ the gyromagnetic ratio, $\Delta$ the DW width, $M_{s}$ the saturation magnetization, and $N$ the demagnetizing factors. The geometry of a rectangular wire yields $N_{y}+N_{z} \approx 1$ and $\frac{N_{y}}{N_{z}}=\frac{T}{W}$, leading to a criterion for WB given by $(\beta-\alpha) \frac{u_{c}}{\gamma \Delta}=2 \pi \alpha M_{s}\left|N_{y}-N_{z}\right| \propto\left|\frac{W-T}{W+T}\right|$.

From the simulation results shown in Fig. 4(a), below $W B$, in all four cases the ratio of DW velocity/spin current velocity $=\beta / \alpha=5$, as expected [Schryer 2003, Mougin 2007]. From Fig. 4(a), WB took place in the interval $u=120 \mathrm{~m} / \mathrm{s}$ to $u=140 \mathrm{~m} / \mathrm{s}$ for all the three rectangular cases, independent of whether the initial geometry was TW or VW. This is consistent with theory [Mougin 2007] since the differences in $\left|N_{y}-N_{x}\right|$ between the three cases is within $14 \%$. However, for the trapezoid wire with tapering of $40 \mathrm{~nm}$, the transition from steady motion to WB happened at a critical spin current velocity of $u=180 \mathrm{~m} / \mathrm{s}$, approximately $30 \%$ higher than in the rectangular wires. After WB, the average domain wall velocity dropped significantly which is consistent with the behavior of rectangular wires [Schryer 2003], although it is worth pointing out that the average velocity calculated within the WB regime is not as accurate as that below WB due to the time-varying velocity during the limited simulation time. 
Fig. 4(b) and (c) shows snapshots of the wall during WB ( $u=180 \mathrm{~m} / \mathrm{s}$ ) in the bottom layer of the rectangular wire with $W=160 \mathrm{~nm}$ and the trapezoid wire with $W_{\min }=140 \mathrm{~nm}$ and $W_{\max }=180 \mathrm{~nm}$, respectively. The configuration changes during WB were similar for both cases, but the trapezoid wire required a longer time to form an antivortex core. The critical spin current velocity for WB caused by this amount of edge taper is about the same as seen in the case of thin rectangular wire with $W=$ $180 \mathrm{~nm}$ made of only one $4 \mathrm{~nm}$ layer. The higher critical current from edge taper is presumed to be caused by the thin edge of the wire impeding the formation of the antivortex, which is necessary for WB; therefore, allowing the DW to remain stable to higher spin current velocities and reach a higher DW velocity.

By raising the taper width to 80 and $120 \mathrm{~nm}$, steady DW motion took place until $u=160 \mathrm{~m} / \mathrm{s}$. However, increasing the spin current velocity beyond this did not lead directly to WB. Instead, the DW underwent what we describe as a vibrational instability. As shown in Fig. 4(d), instead of transforming between TW and VW by generating a vortex core, the DW transformed to a asymmetric TW-like configuration, but was not able to overcome the energy barrier required to form a VW and returned to its original TW configuration. For this reason, when analyzing its velocity over time, instead of oscillating between negative and positive values in the WB regime, the velocity only oscillated between a lower positive value and a higher positive value in the vibrational instability regime. From Fig. 4(a), the average velocity in the vibrational instability regime is about the same as that of the steady motion at $u=160 \mathrm{~m} / \mathrm{s}$ and much higher than that of the WB regime. It is worth noting that this vibrational instability regime may also occur in the $40 \mathrm{~nm}$ tapered wire, but with the step size used in our simulations $(\Delta u=20 \mathrm{~m} / \mathrm{s})$, this may not have been detectable in this wire. With further increase in spin current, a transition from vibrational instability to WB took place at $u=240 \mathrm{~m} / \mathrm{s}$ and $u=280 \mathrm{~m} / \mathrm{s}$ for the $80 \mathrm{~nm}$ tapered wire and $120 \mathrm{~nm}$ tapered wire, respectively.

To explain the existence of the vibrational instability regime, we consider the quasi-1-D model of DW motion. In this model, the spin transfer torque (STT) tilts the magnetization of the center of the DW out of plane and this torque is balanced by the demagnetization field at the surface originating from shape anisotropy. When the current is too high, the demagnetization field is not able to balance the STT and the magnetization is able to precess in the plane normal to the direction of DW motion. However, when the film is tapered, the demagnetizing field varies across the width and the demagnetization field at the edge is still able to balance the STT at a spin current velocity that would cause the thicker central part of the DW to undergo precession. This competition between the edge and the center thus shifts the critical current for WB.

\section{CONCLUSION}

In conclusion, tapering of the edges of magnetic nanowires is predicted to have important effects on both their configuration and their response to a current, delaying the onset of Walker breakdown. Although other methods have been demonstrated to increase the critical current for WB, such as using extremely thin wires, adding periodic current shunt pads [Jang 2012] or patterning comb-like structures [Lewis 2010], the edge taper demonstrated in this paper provides a convenient and easily implemented strategy to tailor the domain wall structure and current-driven dynamics in magnetic wires with relevance to domain wall memory and logic devices.

\section{ACKNOWLEDGMENT}

The authors acknowledge the support of C-SPIN, a STARnet Center of DARPA and MARCO, and the NSF DMR 1419807. Facilities of CMSE and the Nano Structures Laboratory at MIT were used.

\section{REFERENCES}

Allwood D A, Xiong G, Faulkner C C, Atkinson D, Petit D, and Cowburn R P (2005), "Magnetic domain-wall logic," Science, vol. 309, pp. 1688-1692, doi: 10.1126/science.1108813.

Beach G, Tsoi M, and Erskine J (2008), "Current-induced domain wall motion," J. Magn. Magn. Mater., vol. 320, pp. 1272-1281, doi: 10.1016/j.jmmm.2007.12.021.

Berger L (1978), "Low field magnetoresistance and domain drag in ferromagnets," J. Appl. Phys., vol. 49, pp. 2156-2161, doi: 10.1063/1.324716.

Currivan J A, Jang Y, Mascaro M D, Baldo M A, and Ross C A (2012), "Low energy magnetic domain wall logic in short, narrow, ferromagnetic wires," IEEE Magn. Lett., vol. 3, 3000104, doi: 10.1109/LMAG.2012.2188621.

Donahue M J and Porter D G (1999), OOMMF User's Guide. U.S. Department of Commerce, Technology Administration, Nat. Inst. Stand. Technol.

Griffin P B, Plummer J D, and Deal M D, (2000), Silicon VLSI Technology: Fundamentals, Practice, and Modeling. 1st. ed., Englewood Cliffs, NJ, USA: Prentice-Hall.

Jang Y, Mascaro M D, Beach G S D, and Ross C A (2012), "Current-driven domain wall motion in heterostructured ferromagnetic nanowires," Appl. Phys. Lett., vol. 100, 112401, doi: 10.1063/1.3692797.

Kläui M, Vaz C A F, Bland J A C, Heyderman L J, Nolting F, Pavlovska A, Bauer E, Cherifi S, Heun S, and Locatelli A (2004), "Head-to-head domain-wall phase diagram in mesoscopic ring magnets," Appl. Phys. Lett., vol. 85, pp. 56375639, doi: 10.1063/1.1829800.

Lewis E R, Petit D, O'Brien L, Fernandez-Pacheco A, Sampaio J, Jausovec AV, Zeng H T, Read D E, and Cowburn R P (2010), "Fast domain wall motion in magnetic comb structures," Nature Mater., vol. 9, pp. 980-983, doi: 10.1038/nmat2857.

Maranville B B, McMichael R D, Kim S A, Johnson W L, Ross C A, and Cheng J Y (2006), "Characterization of magnetic properties at edges by edge-mode dynamics," J. Appl. Phys., vol. 99, 08C703, doi: 10.1063/1.2167633.

McMichael R D and Donahue M J (1997), "Head to head domain wall structures in thin magnetic strips," IEEE Trans. Magn., vol. 33, pp. 4167-4169, doi: 10.1109/20.619698

Mougin A, Cormier M, Adam J P, Metaxas P J, and Ferré J (2007), "Domain wall mobility, stability and Walker breakdown in magnetic nanowires," Europhys. Lett., vol. 78, 57007, doi: 10.1209/0295-5075/78/57007.

Nakatani Y, Thiaville A, and Miltat J (2005), "Head-to-head domain walls in soft nano-strips: A refined phase diagram," J. Magn. Magn. Mater., vol. 290-291, pp. 750-753, doi: 10.1016/j.jmmm.2004.11.355

Parkin S S P, Hayashi M, and Thomas L (2008), "Magnetic domain-wall racetrack memory," Science, vol. 320, pp. 190-194, doi: 10.1126/science.1145799.

Schryer N L and Walker L R (2003), "The motion of $180^{\circ}$ domain walls in uniform dc magnetic fields," J. Appl. Phys., vol. 45, pp. 5406-5421, doi: $10.1063 / 1.1663252$

Tatara G and Kohno H (2004), "Theory of current-driven domain wall motion: Spin transfer versus momentum transfer," Phys. Rev. Lett., vol. 92, 086601, doi: 10.1103/PhysRevLett.92.086601.

Thiaville A, Nakatani Y, Miltat J, and Suzuki Y (2007), "Micromagnetic understanding of current-driven domain wall motion in patterned nanowires," Europhys. Lett.,vol. 69, pp. 990-996, doi: 10.1209/epl/i2004-10452-6.

Zhang J and Ross C A (2013), "Gold shunt pads as a chirality filter for currentdriven $360^{\circ}$ domain wall motion in a ferromagnetic wire," Appl. Phys. Lett., vol. 103, 162411 doi: 10.1063/1.4825264. 